\title{
Patients with reduced heart rate response to adenosine infusion have low myocardial flow reserve in ${ }^{13} \mathrm{~N}$-ammonia PET studies
}

\author{
Takeshi Tomiyama $^{1}$ Shin-ichiro Kumita ${ }^{1} \cdot K$ eiichi Ishihara ${ }^{2} \cdot$ Masaya Suda $^{2}$. \\ Minoru Sakurai $^{2} \cdot$ Kenta Hakozaki $^{2}$. Hidenobu Hashimoto ${ }^{1} \cdot$ Naoto Takahashi $^{3}$. \\ Hitoshi Takano $^{3}$ - Yasuhiro Kobayashi $^{1} \cdot$ Tomonari Kiriyama $^{1} \cdot$ Yoshimitsu Fukushima $^{1}$. \\ Wataru Shimizu ${ }^{3}$
}

Received: 29 January 2015/Accepted: 30 March 2015/Published online: 7 April 2015

(C) The Author(s) 2015. This article is published with open access at Springerlink.com

\begin{abstract}
To assess the effect of adenosine infusion by evaluating the relationship between heart rate (HR) response to adenosine and myocardial flow reserve (MFR) of remote regions supplied by normal coronary arteries in ${ }^{13} \mathrm{~N}$-ammonia PET. Thirty-one consecutive subjects (20 known coronary artery disease patients, 4 chronic heart failure patients, and 7 normal volunteers) except cases having 3-vessel disease underwent rest and adenosine stress ${ }^{13} \mathrm{~N}$-ammonia myocardial perfusion PET. Semiquantitative, quantitative, and gated analyses were performed. Subjects were divided into two groups with regard to HR response to adenosine. Twenty-two subjects had normal HR response (peak/rest HR $>1.20$ ), while reduced HR response $(\leq 1.20)$ was observed in nine subjects. There were no differences in rest myocardial blood flow (MBF) of remote regions between the groups. Subjects with reduced HR response had significantly lower stress MBF and MFR of remote regions than those with normal HR response (stress MBF: $1.559 \pm 0.517$ vs. $2.279 \pm 0.530$, $p=0.004, \quad$ MFR: $\quad 1.59 \pm 0.36$ vs. $2.35 \pm 0.53$, $p=0.001)$. There were no significant differences between the groups by means of semi-quantitative scoring. Rest and stress ejection fraction (EF) in the reduced HR response group was lower than that in the normal HR response group. In a multiple stepwise regression analysis, HR ratio,
\end{abstract}

Takeshi Tomiyama

tk2@nms.ac.jp

1 Department of Radiology, Nippon Medical School, 1-1-5 Sendagi, Bunkyo-ku, Tokyo 113-8603, Japan

2 Clinical Imaging Center for Healthcare, Nippon Medical School, Tokyo, Japan

3 Department of Cardiovascular Medicine, Nippon Medical School, Tokyo, Japan dyslipidemia, and Brinkman index were identified as predictors of the change in MFR of remote regions. Subjects with reduced HR response to adenosine had lower stress MBF and MFR of remote regions and lower EF. Moreover, HR response was one of the predictors of the change in MFR of remote regions.

Keywords Heart rate response - Adenosine $\cdot$ Myocardial blood flow (MBF) · Myocardial flow reserve (MFR) .

${ }^{13} \mathrm{~N}$-ammonia $\cdot$ Positron emission tomography (PET)

\section{Introduction}

In adenosine stress myocardial perfusion single photon emission computed tomography (SPECT) imaging, reduced blood flow is estimated according to the degree of hyperemia between normal and stenosed coronary arteries. Adenosine induces approximately a four-fold increase in coronary blood flow [1]. Adenosine generally yields an increase in heart rate (HR) and a decrease in blood pressure (BP). Reduced HR response to adenosine or dipyridamole was reported to relation with abnormal perfusion, low ejection fraction (EF), and cardiac autonomic neuropathy [2-5]. Moreover, patients undergoing adenosine stress perfusion SPECT with high rest HR and low peak/rest HR ratio have an increased risk of cardiac death $[6,7]$.

${ }^{13} \mathrm{~N}$-ammonia myocardial perfusion positron emission tomography (PET) is generally performed under pharmacologic stress condition because the half-life of ${ }^{13} \mathrm{~N}-\mathrm{am}$ monia is only about $10 \mathrm{~min}$. Myocardial perfusion PET with ${ }^{13} \mathrm{~N}$-ammonia can evaluate the absolute rest and stress myocardial blood flow (MBF) and myocardial flow reserve (MFR) [8, 9]. Reduced MFR occurs due to coronary stenosis, and microvascular and vascular endothelial 
dysfunctions [10-13]. Stress MBF and MFR should be assessed under the proper adenosine stress test. The aim of this study was to validate heterogeneity of hemodynamic response to adenosine, and to assess the association between hemodynamic response and MFR as well as diagnostic performance of myocardial ischemia in ${ }^{13} \mathrm{~N}$ ammonia PET studies.

\section{Materials and methods}

\section{Study population}

Thirty-one consecutive subjects (22 males, 9 females; mean age $\pm \mathrm{SD}, 66.3 \pm 11.8$ year) underwent rest and adenosine stress ${ }^{13} \mathrm{~N}$-ammonia myocardial perfusion PET. Twenty subjects had known coronary stenosis: 1-vessel stenosis in 8 patients and 2-vessel stenosis in 12 patients. Eleven subjects had no known coronary artery stenosis: seven subjects were normal volunteers, and four subjects underwent PET studies to assess chronic heart failure. Normal volunteers were in good health and had never undergone coronary computed tomography (CT) angiography or coronary angiography (CAG), but some of the subjects had hypertension, dyslipidemia, or diabetes mellitus. Cases with 3-vessel coronary artery disease were excluded because MBF and MFR of all coronary territories were influenced by coronary artery stenosis. Coronary stenosis was defined as $>50 \%$ diameter stenosis shown by CAG. Exclusion criteria with regard to the adenosine test were asthma, pacemaker rhythm, second or third degree atrioventricular block, or sick sinus syndrome. Written informed consent was obtained from all subjects. This study was approved by the local ethics committee and was performed in accordance with the ethical standards of the Declaration of Helsinki.

\section{Data acquisition and adenosine stress protocol}

All subjects had fasted for $>6 \mathrm{~h}$ and had refrained from caffeine-containing beverages and food, smoking, and medications for $24 \mathrm{~h}$ prior to imaging. PET scan was performed on a GEMINI TF-16 (Philips Medical Systems), which is a hybrid time-of-flight (TOF)-PET/CT scanner [14]. PET acquisition was performed in the three-dimensional list mode. The scatter correction was a TOF single scatter simulation algorithm (TOF-SSS), while the attenuation correction was based on rest and stress cardiac CT scans. At rest, approximately $370 \mathrm{MBq}$ of ${ }^{13} \mathrm{~N}$-ammonia was injected into a peripheral vein of all subjects, followed by a $30 \mathrm{~mL}$ saline flush. Dynamic and gated acquisition was initiated just before injection, and was extended for $20 \mathrm{~min}$. Adenosine stress test was performed more than 50 min (5 half-lives) after rest protocol. During this time, ${ }^{13} \mathrm{~N}$ activity at rest had physically decayed to about $3 \%$ of its initial activity. Adenosine was infused at $120 \mu \mathrm{g} / \mathrm{kg} / \mathrm{min}$ for $6 \mathrm{~min}$, according to Japanese clinical conventions. ${ }^{13} \mathrm{~N}$ ammonia and adenosine were infused through the peripheral veins of both the right and left arms (2-route method) to prevent suspending adenosine infusion during administration of ${ }^{13} \mathrm{~N}$-ammonia. No patient exercised during adenosine stress test. ${ }^{13} \mathrm{~N}$-ammonia of the same dose (370 MBq) was injected at the end of the $3 \mathrm{~min}$ of administration. HR, $\mathrm{BP}$, and a 12-lead electrocardiogram (ECG) were obtained every minute during and after adenosine infusion, with continuous ECG monitoring. Twenty-five dynamic frames were reconstructed $(10 \times 5,10 \times 6,1 \times 10,2 \times 30 \mathrm{~s}$, $1 \times 1$, and $1 \times 6 \mathrm{~min}$, for a total of $10 \mathrm{~min}$ ). The reconstruction parameters for TOF-ordered subset expectation maximization (OSEM) were set at three iterations and 33 subsets as optimized for the clinical application.

During stress tests, hemodynamic response to adenosine was recorded. Peak HR was defined as the highest HR during the observation period. HR ratio was calculated by dividing the peak HR by the baseline HR. A reduced HR response to adenosine was considered if the HR ratio was 1.20 or less, according to previous reports [5-7]. Participants were divided into two groups according to HR ratio: normal and reduced HR response groups. Minimum BP was defined as the lowest mean BP during the observation period. Significant ST depression was defined as $\geq 1 \mathrm{~mm}$ of either horizontal or down sloping or $\geq 1.5 \mathrm{~mm}$ of up sloping; STsegment depression was measured $80 \mathrm{~ms}$ after the $\mathrm{J}$ point.

\section{Image interpretation}

Images were re-sliced in the short-axis, as well as in the vertical and horizontal long-axis orientations. Regional ${ }^{13} \mathrm{~N}$ ammonia accumulation was assessed using the American Heart Association (AHA) 17-segment model, and the semiquantitative scoring system of defect severity and extent [15]. A 5-point scale scoring method was used, as follows: 0 , no defect; 1 , mildly reduced; 2 , moderately reduced; 3 , severely reduced; and 4, absent of activity. Image interpretation was performed by two experienced readers. Discordance of scoring was classified by consensus. For each image, a summed rest score (SRS) and a summed stress score (SSS) were calculated by adding the segment scores at rest and stress, respectively. A summed differential score (SDS) was calculated by subtracting the SRS from the SSS.

Quantitative analysis was performed using PMOD software package (version 3.4, PMOD Technologies Ltd., Zurich, Switzerland). Regions of interest were semi-automatically placed on the left ventricular myocardium, and the left and right ventricular blood pools in each slice. The time-activity curves of myocardial and blood pool, 
generated from the dynamic frames, were fitted with the tracer kinetic model (DeGrado 1-compartment model) [16]. Only the first $4 \mathrm{~min}$ of data were used for the curve fitting. The estimated rest and stress MBF were expressed in each segment and territory, while the MFR was calculated by dividing the stress MBF by the rest MBF. MBF and MFR in remote regions supplied by normal coronary arteries were used for statistical analyses: global values for normal coronary disease, mean values of normal 2-territory for 1-vessel disease, and values of normal 1-territory for 2-vessel disease. Rest MBF values modified by rate-pressure product (mean values of 31 subjects: 8800 ) were also calculated. Rest and post-stress end-diastolic volume (EDV), end-systolic volume (ESV), and EF were derived from each image using cardioREPO software (FUJIFILM RI Pharma Co. Ltd,) [17].

\section{Statistics}

All statistical analyses were performed using SPSS version 19 for Windows (SPSS Inc., Chicago, IL). Continuous variables are expressed as mean $\pm \mathrm{SD}$. Categorical variables were compared between normal and reduced HR response groups using Chi square tests. The Wilcoxon rank sum test was used to compare nonparametric variables between the groups. A Pearson's product moment correlation coefficient and a standard linear regression analysis were also used to describe the correlation between the HR ratio and quantitative values. Multiple stepwise regression analysis was used to determine predictors as appropriate. MFR of remote regions was used as dependent variable, and gender, age, body mass index (BMI), hypertension, diabetes mellitus, dyslipidemia, estimated glomerular filtration rate (eGFR), Brinkman index, the number of coronary artery stenosis, symptom, HR ratio (as numerical values), $\Delta \mathrm{BP}$, symptoms and $\mathrm{ST}$ changes during adenosine infusion, rest EDV, rest ESV, and rest EF were the independent variables. A $p$ value $<0.05$ was deemed statistically significant. Moreover, sensitivity, specificity, positive predictive value (PPV), and negative predictive value (NPV) for myocardial ischemia in normal and reduced HR response groups were calculated. Myocardial ischemia was defined if the visual stress score was higher than the rest one for each territory. A gold standard of ischemia was $>50 \%$ stenosis on CAG.

\section{Results}

Twenty-two subjects had normal HR response, while reduced HR response was observed in nine subjects; 5 of 20 coronary artery disease patients, 3 of 4 chronic heart failure patients, and 1 of 7 normal volunteers had reduced HR response. Baseline characteristics of the two groups are shown in Table 1. Subjects with reduced HR response frequently had a smoking habit $(p=0.015)$ and tended to take $\beta$-blocker $(p=0.074)$. There were no differences between the groups in gender, age, BMI, history of hypertension, diabetes mellitus, dyslipidemia, kidney dysfunction, the number of abnormal coronary arteries, and symptoms.

During the adenosine stress test, no patient had any significant adverse reactions, and required a reduction in the dose of adenosine or administration of aminophylline. Hemodynamic responses to adenosine, myocardial perfusion, and gated PET analyses between the groups are shown in Table 2. Average HR increased by 23.3 beats/min in the normal HR response group compared with 9.6 beats/min in the reduced HR response group. The subjects with reduced HR response had a significantly higher baseline HR than those with normal HR response $(73.3 \pm 10.4$ vs. $64.7 \pm 7.6$, $p=0.041$ ). Symptoms (chest pain or chest compression) during adenosine infusion were frequently observed in the normal HR response group $(p=0.036)$. There were no differences in BP and semi-quantitative myocardial perfusion (SRS, SSS, and SDS) between the groups. Stress MBF and MFR of remote regions (supplied by normal coronary arteries) were significantly lower in the reduced HR response group than those in the normal HR response group (stress MBF: $1.559 \pm 0.517$ vs. $2.279 \pm 0.530, p=0.004$, MFR: $1.59 \pm 0.36$ vs. $2.35 \pm 0.53, p=0.001$ ). However, there was no significant difference between the groups with respect to rest $\operatorname{MBF}(p=0.794)$. Rate-pressure product tended to be higher in the reduced HR response group than that in the normal HR response group $(9935 \pm 3147$ vs. $8336 \pm 1583$, $p=0.240$ ). When rest MBF was modified by rate-pressure product, rest MBF and MFR in the reduced HR response group tended to be lower than that in the normal HR response group ( $p=0.090$ and 0.177 , respectively). Rest and stress MBF of stenosed regions between the groups were statistically similar (rest MBF: $1.085 \pm 0.245$ vs. $0.988 \pm 0.126$, $p=0.458$, stress MBF: $1.698 \pm 0.362$ vs. $2.077 \pm 0.598$, $p=0.239$ ), while MFR of stenosed regions in the reduced HR response group was lower than that in the normal HR response group $(1.59 \pm 0.23$ vs. $2.10 \pm 0.50, p=0.050)$. In addition, stress EF in the reduced HR response group was significantly lower than that in the normal HR response group (49.7 \pm 9.6 vs. $60.3 \pm 9.5, p=0.003)$. Rest $\mathrm{EF}$ also tended to be lower in the reduced HR response group $(55.7 \pm 13.1$ vs. $65.2 \pm 8.9, p=0.090)$. Linear regression analyses between HR response and quantitative values are shown in Fig. 1. A good correlation was observed between HR ratio and MFR of remote regions $(r=0.481)$.

In a multiple stepwise regression analysis, MFR of remote regions, gender, age, BMI, hypertension, diabetes mellitus, dyslipidemia, eGFR, Brinkman index, the number of coronary artery stenosis, symptom, HR ratio (as 
Table 1 Baseline

characteristics according to normal or reduced heart rate ratio

\begin{tabular}{|c|c|c|c|}
\hline & Normal HRR $(\mathrm{n}=22)$ & Reduced HRR ( $\mathrm{n}=9$ ) & $p$ value \\
\hline Gender (male) & $14(63.6 \%)$ & $8(88.9 \%)$ & 0.160 \\
\hline Age (y/o) & $65.5 \pm 11.8$ & $68.2 \pm 11.5$ & 0.571 \\
\hline Body mass index $\left(\mathrm{kg} / \mathrm{m}^{2}\right)$ & $23.7 \pm 3.9$ & $22.6 \pm 4.2$ & 0.572 \\
\hline Hypertension & $15(68.2 \%)$ & $7(77.8 \%)$ & 0.593 \\
\hline Diabetes mellitus & $9(40.9 \%)$ & $3(33.3 \%)$ & 0.694 \\
\hline Dyslipidemia & $14(63.6 \%)$ & $6(66.7 \%)$ & 0.873 \\
\hline Hemodialysis & $1(4.5 \%)$ & $2(22.2 \%)$ & 0.131 \\
\hline $\mathrm{eGFR}\left(\mathrm{ml} / \mathrm{min} / 1.73 \mathrm{~m}^{2}\right)$ & $70.3 \pm 21.8$ & $56.3 \pm 31.5$ & 0.499 \\
\hline \multicolumn{4}{|l|}{ Smoking } \\
\hline Present smoker & $2(9.1 \%)$ & $0(0 \%)$ & \\
\hline Past smoker & $7(31.8 \%)$ & $8(88.9 \%)$ & 0.015 \\
\hline Never & $13(59.1 \%)$ & $1(11.1 \%)$ & \\
\hline Brinkman index & $471.4 \pm 726.8$ & $553.3 \pm 403.3$ & 0.157 \\
\hline \multicolumn{4}{|l|}{ Coronary stenosis } \\
\hline 2-vessel & $9(40.9 \%)$ & $3(33.3 \%)$ & \\
\hline 1-vessel & $6(27.3 \%)$ & $2(22.2 \%)$ & 0.801 \\
\hline 0 -vessel & $7(31.8 \%)$ & $4(44.4 \%)$ & \\
\hline Prior PCI & $4(18.2 \%)$ & $1(11.1 \%)$ & 0.627 \\
\hline$\beta$-blocker & $7(31.8 \%)$ & $6(66.7 \%)$ & 0.074 \\
\hline Ca-blocker & $11(50.0 \%)$ & $5(55.6 \%)$ & 0.779 \\
\hline ACE-inh or ARB & $12(54.5 \%)$ & $5(55.6 \%)$ & 0.959 \\
\hline \multicolumn{4}{|l|}{ Symptom } \\
\hline Typical chest pain & $3(13.6 \%)$ & $0(0 \%)$ & \\
\hline Atypical chest pain & $5(22.7 \%)$ & $2(22.2 \%)$ & 0.492 \\
\hline Asymptomatic & $14(63.6 \%)$ & $7(77.8 \%)$ & \\
\hline
\end{tabular}

Subjects with reduced HR response frequently had a smoking habit and tended to take $\beta$-blocker. $H R R$ heart rate response, $e G F R$ estimated glomerular filtration rate, $P C I$ percutaneous coronary intervention, $A C E$-Inh angiotensin-converting enzyme inhibitor, $A R B$ angiotensin receptor blocker numerical values), $\triangle \mathrm{BP}$, symptoms and $\mathrm{ST}$ changes during adenosine infusion, rest EDV, rest ESV, and rest EF were taken into account. HR ratio $(p=0.003)$, dyslipidemia $(p=0.033)$, and Brinkman index $(p=0.045)$ were identified as predictors of the change in MFR of remote regions (Table 3).

Sensitivity, specificity, PPV, and NPV for myocardial ischemia in normal and reduced HR response groups are listed in Table 4. Diagnostic accuracy in reduced HR response group was worse than that in normal HR response group.

\section{Discussion}

Adenosine induces maximal myocardial hyperemia immediately and has a short half-life ( $<10$ s) [18]. Hemodynamic responses during adenosine infusion include a slight increase in HR and a slight decrease in systemic BP. Adenosine induces baroreceptor reflex response to hypotension and directly stimulates the sympathetic nervous system regardless of BP changes, increasing HR [19].
In the present study, 29.0\% (9/31) of all subjects demonstrated reduced HR response during adenosine stress conditions. Even normal coronary subjects (one normal volunteer and three chronic heart failure patients) showed reduced $\mathrm{HR}$ response, suggesting that reduced $\mathrm{HR}$ response was not only dependent on coronary stenosis. The subjects with reduced $\mathrm{HR}$ response in this study were fewer than those reported in previous studies [5, 6]. This is probably because a 2-route method was adopted in this study as the adenosine injection protocol. Using this protocol, adenosine infusion was performed more effectively because suspending adenosine infusion during administration of ${ }^{13} \mathrm{~N}$-ammonia was not necessary.

The subjects with reduced HR response frequently had a smoking habit, the use of $\beta$-blocker, higher rest HR, lower stress MBF and MFR of remote regions, and lower EF in the present study. Smoking habit and the use of $\beta$-blocker might deteriorate the pharmaceutical action of adenosine. Higher rest $\mathrm{HR}$ and lower $\mathrm{EF}$ in the reduced $\mathrm{HR}$ response group were consistent with the findings of previous studies [4-6]. In these studies with comparatively larger populations on 
Table 2 Hemodynamic responses to adenosine, myocardial perfusion, and gated PET analyses in normal and reduced heart rate response (HRR) groups

\begin{tabular}{|c|c|c|c|}
\hline Variable & Normal HRR $(\mathrm{n}=22)$ & Reduced HRR $(\mathrm{n}=9)$ & $p$ value \\
\hline Rest HR (bpm) & $64.7 \pm 7.6$ & $73.3 \pm 10.4$ & 0.041 \\
\hline Peak HR (bpm) & $88.0 \pm 10.0$ & $82.9 \pm 11.5$ & 0.305 \\
\hline Heart rate ratio & $1.37 \pm 0.12$ & $1.13 \pm 0.03$ & $<0.001$ \\
\hline Rest mean BP (mmHg) & $88.1 \pm 11.2$ & $94.9 \pm 12.1$ & 0.207 \\
\hline Minimum mean BP $(\mathrm{mmHg})$ & $79.4 \pm 10.3$ & $87.7 \pm 15.3$ & 0.151 \\
\hline$\Delta \mathrm{BP}$ (minimum-rest) (mmHg) & $-6.5 \pm 8.3$ & $-7.1 \pm 8.4$ & 0.617 \\
\hline Baseline rate-pressure product ${ }^{\mathrm{a}}$ & $8336 \pm 1583$ & $9935 \pm 3147$ & 0.240 \\
\hline Symptoms during adenosine infusion & $8(36.4 \%)$ & $0(0 \%)$ & 0.036 \\
\hline ST change during adenosine infusion & $9(40.9 \%)$ & $3(33.3 \%)$ & 0.694 \\
\hline SRS & $1.7 \pm 3.0$ & $2.6 \pm 4.2$ & 0.834 \\
\hline SSS & $5.5 \pm 7.3$ & $7.9 \pm 7.9$ & 0.386 \\
\hline SDS & $3.8 \pm 5.6$ & $5.3 \pm 6.0$ & 0.372 \\
\hline Rest MBF (ml/min/g) & $0.980 \pm 0.142$ & $0.965 \pm 0.215$ & 0.794 \\
\hline Modified rest $\mathrm{MBF}(\mathrm{ml} / \mathrm{min} / \mathrm{g})^{\mathrm{b}}$ & $1.060 \pm 0.193$ & $0.901 \pm 0.219$ & 0.090 \\
\hline Stress MBF (ml/min/g) & $2.279 \pm 0.530$ & $1.559 \pm 0.517$ & 0.004 \\
\hline MFR & $2.35 \pm 0.53$ & $1.59 \pm 0.36$ & 0.001 \\
\hline Modified MFR ${ }^{\mathrm{c}}$ & $2.20 \pm 0.53$ & $1.83 \pm 0.74$ & 0.177 \\
\hline Rest EDV (ml) & $100.6 \pm 31.1$ & $129.6 \pm 56.4$ & 0.164 \\
\hline Rest ESV (ml) & $36.3 \pm 18.0$ & $64.1 \pm 43.4$ & 0.139 \\
\hline Rest EF (\%) & $65.2 \pm 8.9$ & $55.7 \pm 13.1$ & 0.090 \\
\hline Stress EDV (ml) & $113.0 \pm 36.4$ & $138.4 \pm 56.2$ & 0.231 \\
\hline Stress ESV (ml) & $47.0 \pm 23.8$ & $74.2 \pm 42.4$ & 0.074 \\
\hline Stress EF (\%) & $60.3 \pm 9.5$ & $49.7 \pm 9.6$ & 0.003 \\
\hline$\Delta \mathrm{EDV}$ (stress-rest) (ml) & $12.4 \pm 12.8$ & $8.8 \pm 6.8$ & 0.500 \\
\hline$\Delta \mathrm{ESV}$ (stress-rest) (ml) & $10.7 \pm 9.8$ & $10.1 \pm 9.3$ & 0.896 \\
\hline$\Delta \mathrm{EF}$ (stress-rest) $(\%)$ & $-4.9 \pm 5.2$ & $-5.9 \pm 6.1$ & 0.777 \\
\hline
\end{tabular}

$B P$ blood pressure, $S R S$ summed rest score, $S S S$ summed stress score, $S D S$ summed differential score, $M B F$ myocardial blood flow, MFR myocardial flow reserve, EDV end-diastolic volume, ESV end-systolic volume, $E F$ ejection fraction

${ }^{\mathrm{a}}$ Rate-pressure product $=\mathrm{HR} \times$ systolic $\mathrm{BP}$

${ }^{\mathrm{b}}$ Modified rest $\mathrm{MBF}=$ rest $\mathrm{MBF} \times(8800 /$ rate-pressure product $)$

${ }^{c}$ Modified MFR $=$ stress $\mathrm{MBF} /$ modified rest $\mathrm{MBF}$
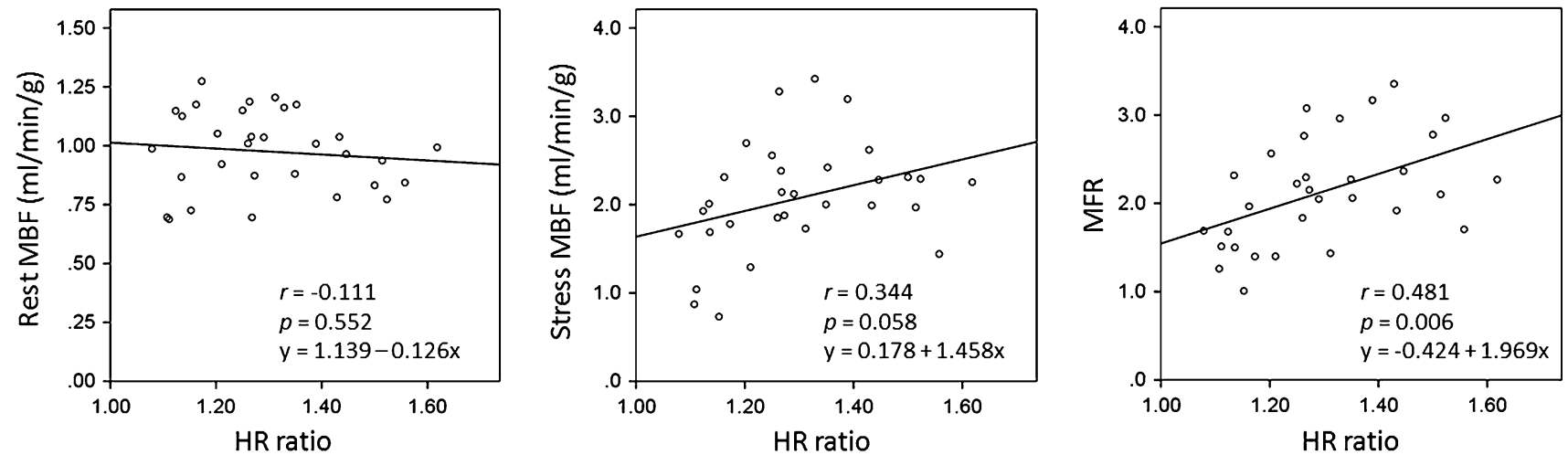

Fig. 1 Linear regression analyses between heart rate (HR) ratio and quantitative values of remote regions. There was a good correlation between HR response to adenosine and myocardial flow reserve of remote regions 
Table 3 Multiple stepwise regression analysis with stepping method criteria of probability of $F$ to enter $<0.05$ and to remove $>0.10$

\begin{tabular}{lcc}
\hline Variable & Standardized coefficients $(\beta)$ & $p$ value \\
\hline HR ratio & 0.480 & 0.003 \\
Dyslipidemia & -0.329 & 0.033 \\
Brinkman index & -0.308 & 0.045 \\
\hline
\end{tabular}

Independent variables $(p<0.05)$ are listed

Table 4 Diagnostic ability of visual assessment in ${ }^{13} \mathrm{~N}$-ammonia PET studies for myocardial ischemia in normal and reduced HR response groups $(>50 \%$ stenosis on CAG was considered as a gold standard)

\begin{tabular}{lllll}
\hline & Sensitivity & Specificity & PPV & NPV \\
\hline Normal HRR & $70.8 \%$ & $92.9 \%$ & $85.0 \%$ & $84.8 \%$ \\
$(\mathrm{n}=66$ territories $)$ & $(17 / 24)$ & $(39 / 42)$ & $(17 / 20)$ & $(39 / 46)$ \\
Reduced HRR & $62.5 \%$ & $84.2 \%$ & $62.5 \%$ & $84.2 \%$ \\
$(\mathrm{n}=27$ territories) & $(5 / 8)$ & $(16 / 19)$ & $(5 / 8)$ & $(16 / 19)$ \\
\hline
\end{tabular}

$H R R$ heart rate response, $P P V$ positive predictive value, $N P V$ negative predictive value

myocardial perfusion SPECT, reduced HR response was related to SRS and SSS, and not to SDS; however, the present preliminary study did not demonstrate differences of SRS, SSS, and SDS between the groups. Regardless of similar semi-quantitative perfusion scores, stress MBF and MFR of remote regions were lower in the reduced HR response group than those in the normal HR response group. It is considered that lower stress MBF and MFR of remote regions with reduced HR response were associated with inadequate reaction to adenosine infusion. When rest MBF was modified by ratepressure product, rest MBF and MFR tended to be lower in the reduced HR response group than those in the normal HR response group. Rate-pressure product correction made differences of MFR between the groups smaller and less significant. The subjects with reduced HR response were supposed to have increased cardiac work and higher level of intrinsic adenosine at rest. Extrinsic adenosine response could be insufficient because intrinsic adenosine has already made coronary arteries dilated and coronary arteries did not have sufficient vasodilator capacity. Moreover, because stress MBF was significant lower in the reduced HR response group, non-responders whom adenosine did not have work well regardless of rate-pressure product were thought to truly exist. Therefore, reduced MFR could reflect different hemodynamics between subjects with normal and reduced HR response. For normal HR response subjects, reduced MFR represents coronary stenosis and/or microvascular and endothelial dysfunctions. On the other hand, reduced MFR means insufficient adenosine response in addition to the above (coronary stenosis and/or microvascular and endothelial dysfunctions) for reduced $\mathrm{HR}$ response subjects. Then, absolute (non-corrected) rest MBF and MFR were essential for accurate stratification of reduced MFR. Because stress MBF and MFR of stenosed regions were influenced by coronary stenosis in addition to adenosine response and microvascular and endothelial dysfunctions, quantitative values of remote regions were more useful when adenosine response was assessed. Differences of stress MBF and MFR between the groups were more evident in remote regions than those in stenosed regions.

In addition, the subjects with reduced HR response did not have symptoms during adenosine infusion in this study. That is, non-responders to adenosine had lower HR response and lesser symptoms during adenosine infusion, and lower MFR of remote regions. Moreover, a good correlation between HR response and MFR was indicated by linear regression analysis. To our knowledge, the present study was the first to investigate the association between reduced HR response to adenosine and quantitative values on ${ }^{13} \mathrm{~N}$-ammonia PET studies. Further studies measuring blood caffeine levels are desired. A part of the cause of inadequate response to adenosine can be revealed by measuring blood caffeine levels.

MFR of remote regions is changed by microvascular and vascular endothelial dysfunctions besides the reaction to adenosine [10-13]. A multiple stepwise regression analysis revealed that $\mathrm{HR}$ response to adenosine was an independent predictor of the change in MFR of remote regions, similarly to dyslipidemia and smoking (microvascular and vascular endothelial dysfunctions).

It has previously been reported that the ability to detect coronary artery disease and the diagnostic accuracy of adenosine myocardial perfusion imaging are not affected by variations of hemodynamic response [20, 21]. However, because patients with reduced HR response have lower MFR of remote regions, dilatation of coronary artery was certainly also diminished, leading to underestimation of scar or ischemia. In the present study, diagnostic accuracy of visual assessment for myocardial ischemia in the reduced HR response group was lower than that in the normal HR response group. Low sensitivity in the reduced HR response group was probably due to inadequate effectiveness of adenosine infusion. This deteriorated pharmaceutical action also might yield inhomogeneous myocardial accumulation under stress condition, resulting in reduced specificity. Further studies including larger population and setting functional flow reserve (FFR) as a gold standard would be necessary for validation.

\section{Conclusions}

The subjects with reduced HR response to adenosine infusion had lower stress MBF and MFR of remote regions and lower EF. HR response and MFR of remote regions 
correlated well, and HR response was one of the predictors of the change in MFR. Therefore, careful consideration should be taken into for visual and quantitative analyses of these patients.

Conflict of interest The authors declare that they have no conflict of interest.

Ethical standard All procedures performed in studies involving human participants were in accordance with the ethical standards of the institutional and/or national research committee and with the 1964 Helsinki declaration and its later amendments of comparable ethical standards.

Informed consent Informed consent was obtained from all individual participants included in the study.

Open Access This article is distributed under the terms of the Creative Commons Attribution 4.0 International License (http:// creativecommons.org/licenses/by/4.0/), which permits unrestricted use, distribution, and reproduction in any medium, provided you give appropriate credit to the original author(s) and the source, provide a link to the Creative Commons license, and indicate if changes were made.

\section{References}

1. Chan SY, Brunken RC, Czernin J, Porenta G, Kuhle W, Krivokapich J et al (1992) Comparison of maximal myocardial blood flow during adenosine infusion with that of intravenous dipyridamole in normal men. J Am Coll Cardiol 20:979-985

2. Vashist A, Heller EN, Blum S, Brown EJ, Bhalodkar NC (2002) Association of heart rate response with scan and left ventricular function on adenosine myocardial perfusion imaging. Am J Cardiol 89:174-177

3. Bravo PE, Hage FG, Woodham RM, Heo J, Iskandrian AE (2008) Heart rate response to adenosine in patients with diabetes mellitus and normal myocardial perfusion imaging. Am J Cardiol 102:1103-1106

4. de Souza Leão Lima R, Machado L, Azevedo AB, De Lorenzo A (2011) Predictors of abnormal heart rate response to dipyridamole in patients undergoing myocardial perfusion SPECT. Ann Nucl Med 25:7-11

5. Gorur GD, Ciftci EA, Kozdag G, Isgoren S, Oc MA, Haksal C et al (2012) Reduced heart rate response to dipyridamole in patients undergoing myocardial perfusion SPECT. Ann Nucl Med 26:609-615

6. Mathur S, Shah AR, Ahlberg AW, Katten DM, Heller GV (2010) Blunted heart rate response as a predictor of cardiac death in patients undergoing vasodilator stress technetium- $99 \mathrm{~m}$ sestamibi gated SPECT myocardial perfusion imaging. J Nucl Cardiol $17: 617-624$

7. Abidov A, Hachamovitch R, Hayes SW, Ng CK, Cohen I, Friedman JD et al (2003) Prognostic impact of hemodynamic response to adenosine in patients older than age 55 years undergoing vasodilator stress myocardial perfusion study. Circulation 107:2894-2899

8. Uren NG, Melin JA, De Bruyne B, Wijns W, Baudhuin T, Camici PG (1994) Relation between myocardial blood flow and the severity of coronary-artery stenosis. N Engl J Med 330:1782-1788

9. Muzik O, Duvernoy C, Beanlands RS, Sawada S, Dayanikli F, Wolfe ER Jr et al (1998) Assessment of diagnostic performance of quantitative flow measurements in normal subjects and patients with angiographically documented coronary artery disease by means of nitrogen-13 ammonia and positron emission tomography. J Am Coll Cardiol 31:534-540

10. Alexánderson E, Jácome R, Jiménez-Santos M, Ochoa JM, Romero E, Cabral MA et al (2012) Evaluation of the endothelial function in hypertensive patients with ${ }^{13} \mathrm{~N}$-ammonia PET. J Nucl Cardiol 19:979-986

11. Prior JO, Quiñones MJ, Hernandez-Pampaloni M, Facta AD, Schindler TH, Sayre JW et al (2005) Coronary circulatory dysfunction in insulin resistance, impaired glucose tolerance, and type 2 diabetes mellitus. Circulation 111:2291-2298

12. Yokoyama I, Ohtake T, Momomura S, Nishikawa J, Sasaki Y, Omata M (1996) Reduced coronary flow reserve in hypercholesterolemic patients without overt coronary stenosis. Circulation 94:3232-3238

13. Campisi R, Czernin J, Schöder H, Sayre JW, Marengo FD, Phelps ME et al (1998) Effects of long-term smoking on myocardial blood flow, coronary vasomotion, and vasodilator capacity. Circulation 98:119-125

14. Surti S, Kuhn A, Werner ME, Perkins AE, Kolthammer J, Karp JS (2007) Performance of Philips Gemini TF PET/CT scanner with special consideration for its time-of-flight imaging capabilities. J Nucl Med 48:471-480

15. Cerqueira MD, Weissman NJ, Dilsizian V, Jacobs AK, Kaul S, Laskey WK et al (2002) Standardized myocardial segmentation and nomenclature for tomographic imaging of the heart. A statement for healthcare professionals from the Cardiac Imaging Committee of the Council on Clinical Cardiology of the American Heart Association. Int J Cardiovasc Imaging 18:539-542

16. DeGrado TR, Hanson MW, Turkington TG, Delong DM, Brezinski DA, Vallée JP et al (1996) Estimation of myocardial blood flow for longitudinal studies with 13N-labeled ammonia and positron emission tomography. J Nucl Cardiol 3:494-507

17. Nakajima K, Okuda K, Nyström K, Richter J, Minarik D, Wakabayashi $\mathrm{H}$ et al (2013) Improved quantification of small hearts for gated myocardial perfusion imaging. Eur J Nucl Med Mol Imaging 40:1163-1170

18. Cerqueira MD, Verani MS, Schwaiger M, Heo J, Iskandrian AS (1994) Safety profile of adenosine stress perfusion imaging: results from the Adenoscan Multicenter Trial Registry. J Am Coll Cardiol 23:384-389

19. Dhalla AK, Wong MY, Wang WQ, Biaggioni I, Belardinelli L (2006) Tachycardia caused by A2A adenosine receptor agonists is mediated by direct sympathoexcitation in awake rats. J Pharmacol Exp Ther 316:695-702

20. Aksut SV, Pancholy S, Cassel D, Cave V, Heo J, Iskandrian AS (1995) Results of adenosine single photon emission computed tomography thallium-201 imaging in hemodynamic nonresponders. Am Heart J 130:67-70

21. Amanullah AM, Berman DS, Kiat H, Friedman JD (1997) Usefulness of hemodynamic changes during adenosine infusion in predicting the diagnostic accuracy of adenosine technetium- $99 \mathrm{~m}$ sestamibi single-photon emission computed tomography (SPECT). Am J Cardiol 79:1319-1322 